\title{
Detection and Damping of Thermal-Acoustic Oscillations in Low-Temperature Measurements
}

\author{
David A. Ditmars and George T. Furukawa
}

(June 8, 1964)

\begin{abstract}
The spontaneous thermal-acoustic oscillations in nonisothermal columns of helium, hydrogen, nitrogen, argon, and oxygen gas have been investigated. The pressure fluctuations were measured with a barium titanate transducer and the particle displacements observed by a smoke technique. The observed pressure oscillations were often nonsymmetrical and of sufficient amplitude to indicate the necessity for a nonlinear approach in a theoretical treatment of the oscillations. An adjustable "Helmholtz resonator" attached at the warm end of the oscillating column was found to damp completely the oscillations and eliminate the energy transfer which they caused.
\end{abstract}

\section{Introduction}

Spontaneous pressure oscillations in gaseous columns, along which there is a temperature gradient, often hamper cryogenic work. An excellent review of this oscillation phenomenon has been given by Wexler [6]. ${ }^{1}$ While there are at least three reports of such oscillations having been used to advantage $[1,2,3]$, they are most often undesirable. They can introduce errors in vapor-pressure measurements and sometimes make the measurements impossible to obtain $[1,4]$. Frequently, the oscillations cause a large uncontrollable energy transfer from hot to cold regions, making calorimetric measurements difficult or impossible.

Experience gained with a calorimeter at the National Bureau of Standards confirmed Wexler's observation [6] that the energy transfer due to gas oscillation from the room temperature region to the $4^{\circ} \mathrm{K}$ region can be as much as 1000 times greater than the energy transfer by thermal conduction. It was decided to investigate the possibility of damping the oscillations by some device added externally.

Experiments with a simple auxiliary apparatus showed this solution to be promising. This apparatus was then used to investigate further the factors affecting the cause and suppression of the oscillations. In the course of this investigation, an adjustable "Helmholtz resonator" attached at the warm column end proved to be a reliable and effective damper.

\section{Apparatus}

The auxiliary apparatus shown in figure 1 consists basically of a tube $\mathrm{T}$ extending down into a Dewar containing a cryogenic liquid boiling at atmospheric pressure. The lower end of the tube $T$ was always open and the upper end was closed by a pressure

1 Figures in brackets indicate the literature references at the end of this paper. transducer and a device used to suppress the gaseous oscillations.

The Dewar, of two-liter capacity, was generally used with less than a liter of liquid. When the liquid was helium, the Dewar was immersed in a liquid nitrogen bath.

Temperatures along the tube $\mathrm{T}$ were occasionally measured with thermocouples. Typical temperature gradients along the tube ranged from 1 to $2{ }^{\circ} \mathrm{C}$ per $\mathrm{mm}$ when the Dewar contained 1 liter of liquid. The only means of changing the temperature gradient along the tube was to add or withdraw cryogenic liquid in the Dewar, keeping the same apparatus dimensions.

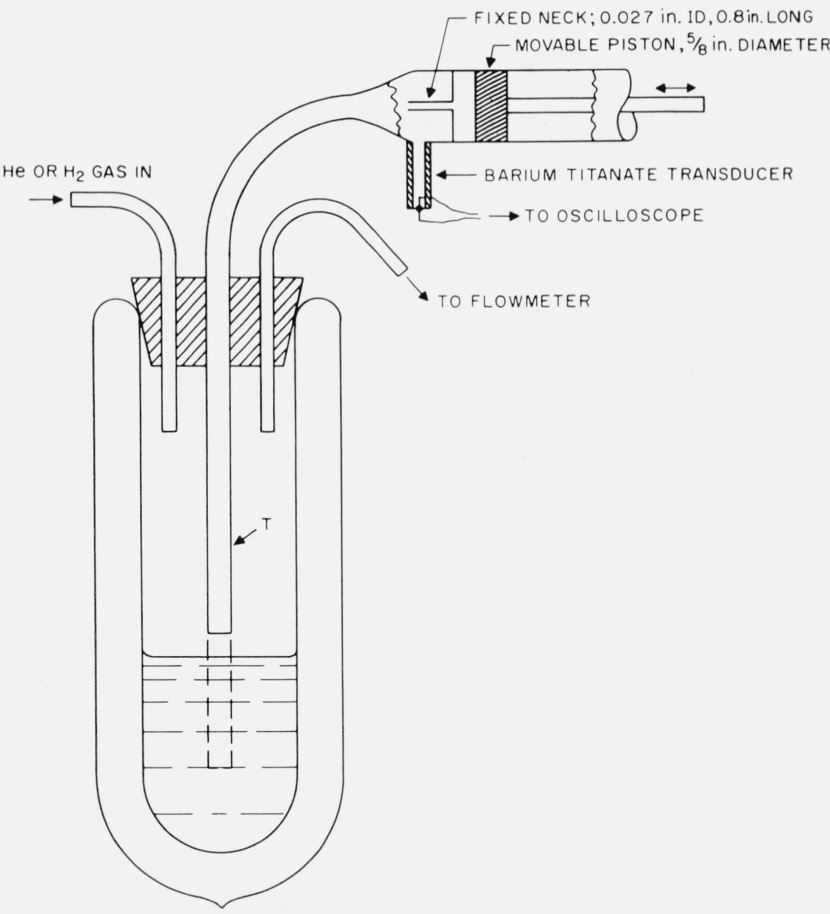

Figure 1. Auxiliary apparatus for studying thermal-acoustic oscillations. 
A barium titanate tube, cemented onto the upper end of $\mathrm{T}$, served as a pressure transducer. This transducer ( 2 in. long, $3 / 8$ in. o.d. with a $1 / 16$ in. wall) was silvered on the inside and outside and polarized through the wall thickness. A static pressure calibration showed that the transducer had a sensitivity of $7 \mathrm{mV} /$ torr $^{2}$ differential pressure across its walls. This potential was measured with a vibrating reed electrometer and was a linear function of pressure in the range covered, 0 to 30 torr. The sensitivity should be independent of frequency at the low frequencies involved in these experiments (less than $60 \mathrm{~Hz}$ ). The transducer output was fed directly to a sensitive oscilloscope. Because of the low impedance of the oscilloscope, the trace, at low frequencies (2 to $10 \mathrm{~Hz}$ ), represented more nearly the time derivative of the average pressure fluctuations inside the transducer than the pressure itself. Additional circuitry or a dynamic calibration of the transducer-oscilloscope system would be necessary to interpret the trace directly as a pressure at low frequencies.

Some direct visual observations of the wave motion were made when ' $\mathrm{T}$ was a glass tube by introducing a smoke of finely divided $\mathrm{TiO}_{2}$ particles into the tube. In the case of oscillations over liquid helium, that part of the energy dissipated in the liquid by the oscillating gas column was measured by passing the gas evolved from the Dewar through a flowmeter.

Pressure and net mass flow of gas in the tube were not controlled in these experiments. The mean pressure in the tube was always within a few torr of atmospheric and the net gas flow in the tube was zero. This is not to imply that oscillations occur only under these conditions. For instance, in the calorimeter referred to above, oscillations have been detected at pressures below 400 torr and also in tubes through which a net helium gas flow existed.

\section{Results}

\subsection{Generation and Observation of Oscillations}

The literature contains only references to oscillations in helium or hydrogen gas columns over baths of liquid helium or hydrogen, respectively. The present work has shown the phenomenon to be of a more general nature. For instance, gaseous columns can also be made to oscillate over baths of liquid nitrogen or oxygen. Columns of helium, hydrogen, nitrogen, argon, or oxygen gas were readily made to oscillate over a liquid oxygen bath. Evaporation caused by oscillation energy transfer to nitrogen or oxygen baths in the present experiments was small and could not be distinguished from evaporation due to other sources of heat leak to the baths. Two conditions had to be met, however, for the oscillations to occur with the higher temperature baths: (a) The open tube end had to extend several tube diameters beneath the bath surface, and (b) the Dewar volume above the surface had to be flooded with helium or hydrogen gas. This

\footnotetext{
21 torr $=1 / 760$ normal atmosphere $=133.322 \mathrm{~N} / \mathrm{m}^{2}$.
}

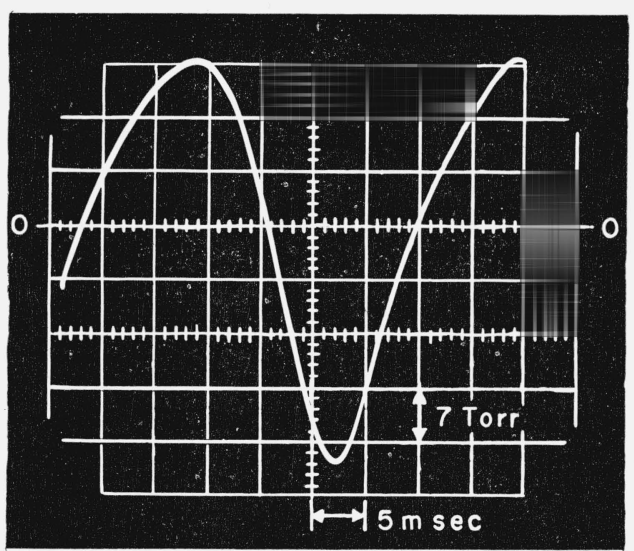

Figure 2. Pressure oscillation at closed end of He gas column. Open end $1.5 \mathrm{~cm}$ above liquid He surface. " $O$ " indicates sweep position without oscillation.

is in distinction to helium or hydrogen gas columns which oscillate in tubes whose open end is either above or below the surface of a liquid helium or hydrogen bath respectively. Probably the most important effect of the flooding was the increase in the surface heat-transfer coefficient [14] at the exterior tube wall. This also altered the bath composition and the tube temperature gradient. Experiments performed, in which these latter effects were eliminated or produced independently of any change in the heat transfer coefficient, showed that helium or hydrogen gas had to be present for oscillations to be sustained.

To obtain a column of a gas other than that of the bath species, the tube T, removed from the Dewar, was first flushed by introducing the desired gas through an opening in the upper tube end. The tube was then lowered into the Dewar until its lower (open) end was beneath the bath surfice and the gas flow gradually reduced to zero. This procedure was followed, for instance, to obtain a column of helium gas above liquid nitrogen. The amplitude and period of the ensuing oscillation were recorded as soon as they reached stable values in order to minimize the effect of contamination of the helium gas column with nitrogen. The observed frequency was always several times that of a nitrogen gas column oscillating under the same condition-as much as eight times in one instance. By way of comparison, a similar gas column resonating in its lowest acoustic mode with helium would normally exhibit a frequency which is about three times that when resonating with nitrogen in the same mode. A helium column oscillating with the open tube end below the bath surface displayed approximately equal frequencies with baths of helium or nitrogen though the amplitude over the helium bath was several times that over the nitrogen bath.

At low amplitudes the pressure oscillations appeared sinusoidal, but with either increasing amplitude or increasing temperature gradient, a nonsymmetrical distortion appeared (fig. 2). The time between a pressure maximum and the following pressure minimum exceeded half the period. 
Using the smoke technique referred to above, observations were made with a glass tube of $2 \mathrm{~mm}$ i.d. and $0.8 \mathrm{~mm}$ wall thickness, containing a nitrogen gas column oscillating at about $5 \mathrm{~Hz}$ over a liquid nitrogen bath. No displacement nodes were observed other than at the closed end. The displacement amplitude of the smoke particles increased monotonically along the tube length and was as great as $\pm 8 \mathrm{~mm}$ near the open (cold) tube end. The amplitude of this oscillation was 5 to 10 times less than typical amplitudes of helium gas oscillations over liquid helium in which a large energy transfer occurred. When direct observation of the liquid meniscus in the tube $\mathrm{T}$ was possible (i.e., with glass tubes), the mean position of the meniscus was below the bath level, indicating a net positive pressure within the tube during oscillation.

\subsection{Damping the Oscillations}

Reported attempts to damp or eliminate thermalacoustic oscillations fall into three main categories:

(1) Changes in apparatus geometry such as employing tubes of greater diameter or closing tubes at the cold end when possible $[2,7]$.

(2) Insertion of objects such as wire, wire mesh or knotted threads into the oscillating column $[5,8,13]$.

(3) Thermal isolation of the oscillating column $[2,4]$. None of these methods was practical to apply to the calorimeter referred to above. It was possible to control the oscillations in the auxiliary apparatus (fig. 1) at any desired amplitude or to eliminate them completely with an adjustable Helmholtz resonator attached at the warm end of the oscillating column. The same technique permitted the control of the oscillations in the calorimeter. The effect of the resonator dimensions on its efficiency as a damper was then investigated using the auxiliary apparatus.

Resonator neck diameters from 0.014 in. to 0.058 in. and lengths up to 2 in. were tried. The neck dimensions were found to be critical and the best results were obtained with the neck dimensions indicated in figure 1. Necks whose diameters or lengths varied from the indicated neck dimensions by as little as a factor of two provided poor damping. Oscillations in helium and nitrogen gas columns ranging in frequency from 2 to $60 \mathrm{~Hz}$ and causing heat leaks of up to $7 \mathrm{~W}$ were successfully damped with the resonator. A typical set of damping curves obtained for helium gas oscillating over liquid helium is given in figure 3. The distance of the tube end above the liquid surface was kept constant along any curve. Larger values of the relative pressure amplitude correspond to lesser values for this distance. These curves varied considerably in shape with different oscillating gas columns and different neck dimensions. They exhibited, however, the common characteristic of a continual decrease in pressure amplitude (and associated energy transfer) with increasing piston extension.

Piston extensions up to three times the minimum length required for complete damping were investigated and in no instance did the oscillation ever reappear. No correlation was discovered between

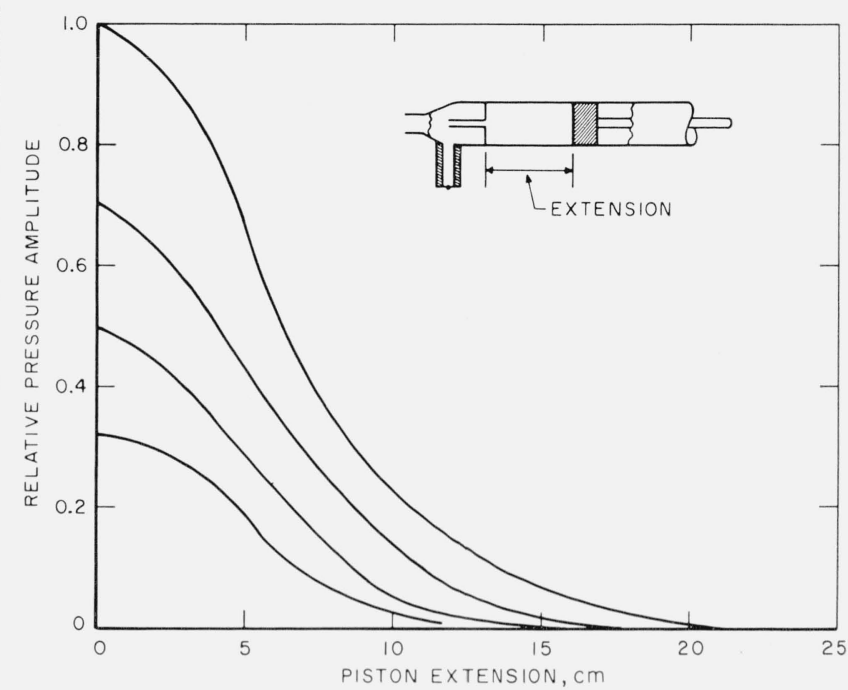

FigurE 3. Effect of piston extension on pressure amplitude. Pressure amplitude increased by decreasing distance between cold tube end and
liquid surface.

the piston diameter and the minimum piston extension required to damp an oscillation of given amplitude; neither was the natural acoustic frequency of the damper related in any obvious way to its effectiveness as a damper. Piston diameters from $3 / 8 \mathrm{in}$. to $1 \mathrm{in}$. have been used successfully at extensions not exceeding $50 \mathrm{~cm}$ to damp oscillations in the entire amplitude and frequency range attainable in the auxiliary apparatus with the combinations of gas columns and liquid baths mentioned earlier.

The damper illustrated in figure 1, together with the oscillating column, constitutes a variable volume svstem. Equivalent results were also obtained by fixing the position of the piston and moving the neck assembly. In this case, the neck was mounted on a thin piston which was attached to a rod extending through a seal in the fixed piston.

In one series of experiments a porous tissue plug was substituted for the neck assembly. The damping of some oscillations was possible with this arrangement.

\section{Discussion}

The neck assembly is the vital part of the damper shown in figure 1. Since oscillations cannot be sustained in a tube open at the warm end, one could, in principle, attach a large closed volume onto the end of any tube to simulate an open end condition and thus damp the oscillations without use of the neck assembly. In practice, the volume required is often rather large and cumbersome. Damper experiments performed with the neck assembly removed have shown that the volume changes alone caused by retraction of the piston have little effect on the oscillation amplitude.

The resonator damper has several advantages. No modification of apparatus design in the cold region and no obstruction in the oscillating tube is required. This is an important consideration if a pumping tube is oscillating. Connections can be 
provided so that the damper can be removed and it can be designed to be vacuum or pressure tight if the gas column is oscillating at a mean pressure considerably different from atmospheric. In the application to the above calorimeter, the piston was designed to be vacuum tight.

The coupling between the damper and oscillating system is not fully understood. Several workers $[9,10]$ have investigated the nonlinear impedance of a Helmholtz resonator at large amplitudes and have tried to correlate it with energy dissipation in vortices near the resonator neck. The flow pattern near the neck of a damper used in the present experiments was observed by the smoke technique and appeared to be a pulsating jet similar to those described in [9].

A number of variables, probably not all independent of one another, determine whether or not a given system will perform sustained oscillations and if so, what the wave form and associated energy transfer will be. With a given temperature distribution throughout the system, the essential requirements seem to be that the gas column have a diameter small enough (usually less than $1 / 2$ in.), that the cold tube end be open to a volume whose characteristic dimensions are large compared to the tube diameter, that the warm end be terminated with a high acoustic impedance, and that the tube be in good thermal contact with its surroundings. This contact need not be due to a conducting gas but can be provided by metal contact with heat sources or sinks along the tube length as was observed in the above calorimeter.

Kramers [15] has attempted to treat the gas oscillations analytically assuming small amplitudes of vibration. This linear approach did not account for the observed phenomena, a result which is plausible considering the oscillation amplitudes encountered in the present work. The possible existence of such oscillating states of a thermal system has been treated in some detail from an energy stand- point by Milne [11]. Milne's theory predicts periodic fluctuations with just the asymmetry observed in these experiments. Squire [12] has speculated that the occurrence of the Boyle temperature in the oscillating column may play a role in initiating the oscillations. Since a column of helium gas readily oscillates over liquid nitrogen, this evidently need not be a decisive role.

Since thermal-acoustic oscillations so often hamper low temperature experiments, the need for a fuller understanding of them is great. It is felt that an apparatus specifically designed to reproduce known temperature gradients would be valuable in future experimental investigation.

\section{References}

[1] K. W. Taconis, J. J. M. Beenakker, A. O. C. Nier, and L. T. Aldrich, Physica 15, 733 (1949).

[2] J. R. Clement, Proc. 1954 Cryogenic Engineering Conf. (1954).

[3] D. Ter Harr, Proc. Paris Conference on Low Temperature Physics, p. 347 (1955). (See appended discussion.)

[4] F. E. Hoare and J. E. Zimmerman, Rev. Sci. Instr. 30, 184 (1959)

[5] O. V. Lounasmaa, Ann. Univ. Turku, A1, No. 39 (1959).

[6] A. Wexler; in F. E. Hoare, L. C. Jackson, and N. Kurti (Eds.), Experimental Cryophysies, p. 155 (Butterworths, London, 1961).

[7] A. Van Itterbeek and W. H. Keesom, Comm. Leiden $209 \mathrm{C}$.

[8] R. B. Scott, Cryogenic Engineering, p. 118 (D. Van Nostrand Co., Inc., 1959).

[9] U. Ingård and S. Labate, J. Acoust. Soc. Am. 30, 211 (1950).

[10] F. Barthel, Frequenz 12, 72 (1958).

[11] E. A. Milne, Quart. Journ. of App. Math. 4, 258 (1933).

[12] C. F. Squire, Low Temperature Physics, p. 23 (McGrawHill Book Co., Inc., New York, N.Y., 1953).

[13] H. Plumb, private communication.

[14] M. Jakob, Heat Transfer, I (John Wiley \& Sons, Inc., New York, N.Y., 1949).

[15] H. A. Kramers, Physica 15, 971 (1949).

(Paper 69C1-183) 